\title{
Long-pulse Plasma Discharges by Upgraded ECH System in the LHD
}

\author{
Y. Yoshimura ${ }^{1}$, H. Kasahara ${ }^{1}$, K. Nagasaki ${ }^{2}$, M. Tokitani ${ }^{1}$, N. Ashikawa ${ }^{1}$, Y. Ueda ${ }^{3}$, S. Ito ${ }^{1}$, S. Kubo ${ }^{1}$, T. Shimozuma $^{1}$,

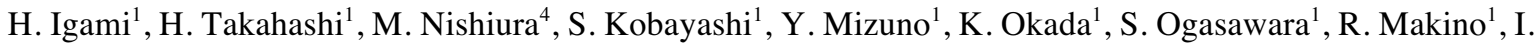

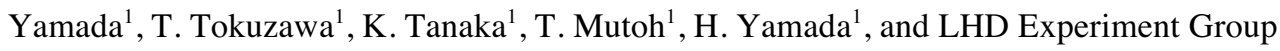 \\ ${ }^{1}$ National Institute for Fusion Science, Toki, Gifu 509-5292, Japan \\ ${ }^{2}$ Institute of Advanced Energy, Kyoto University, Uji, Kyoto 611-0011, Japan \\ ${ }^{3}$ Graduate School of Engineering, Osaka University, Suita, Osaka 565-0871, Japan \\ ${ }^{4}$ Graduate School of Frontier Sciences, The University of Tokyo, Kashiwa, Chiba 277-8561, Japan
}

\begin{abstract}
Until 2009, three high-power, over 1MW each, 77GHz gyrotrons have been installed and applied to the LHD experiment. In addition, a $154 \mathrm{GHz}$ gyrotron of $1 \mathrm{MW}$ was installed in 2012 . The $77 \mathrm{GHz}$ gyrotrons suffer gradual increases of internal pressure during long-pulse operation delivering power to LHD. To mitigate the problem, quasi-steady operation by combination of on-off operations of the $77 \mathrm{GHz}$ gyrotrons was performed. Applying two $77 \mathrm{GHz}$ gyrotrons alternately with intervals of two minutes and an $84 \mathrm{GHz}$ gyrotron continuously, a $30 \mathrm{~min}$. long-pulse discharge with the line average electron density $n_{\text {e_ave }}$ of $0.7 \times 10^{19} \mathrm{~m}^{-3}$ and the central electron temperature $T_{\mathrm{e} 0}$ of $1.7 \mathrm{keV}$ was achieved by the time average injection power $P_{\text {inj }}$ of $260 \mathrm{~kW}$ in 2012, showing significant progress in sustained density from the former $65 \mathrm{~min}$. discharge with $n_{\mathrm{e} \_ \text {ave }}$ of $0.15 \times 10^{19} \mathrm{~m}^{-3}$ and $T_{\mathrm{e} 0}$ of $1.7 \mathrm{keV}$ by $P_{\text {inj }}$ of $110 \mathrm{~kW}$ of $84 \mathrm{GHz}$ wave. In 2013 , one of the $77 \mathrm{GHz}$ gyrotron was improved to furnish a sub-window to remove stray radiation inside the tube. And the new $154 \mathrm{GHz}$ gyrotron was applied to the long-pulse discharge experiment. Using three gyrotrons: 154, the improved and an existing $77 \mathrm{GHz}$ with $P_{\text {inj }}$ of $340 \mathrm{~kW}$ in total, higher temperature plasma having steep temperature gradient typical for internal transport barrier, with $n_{\mathrm{e}_{\_} \text {ave }}$ of $1.1 \times 10^{19} \mathrm{~m}^{-3}$ and $T_{\mathrm{e} 0}$ of $3.5 \mathrm{keV}$ was quite stably sustained for $325 \mathrm{~s}$.
\end{abstract}

\section{Introduction}

The Large Helical Device (LHD) [1] in the National Institute for Fusion Science (NIFS) furnishes superconducting coils and has a great advantage in stable and long-pulse plasma sustainment. In contrast to tokamaks, the magnetic field configuration for plasma confinement in the LHD is completely generated by superconducting helical and poloidal coils so that excitation of toroidal plasma current is not required. Therefore, the LHD is suitable for performing investigations on subjects such as heat removal and plasma-wall interaction, which require long-pulse discharges without the difficulty of plasma current sustainment. Those investigations are necessary and profitable for future steady-state operation up to $1000 \mathrm{~s}$ planned in ITER. Intensive studies on long-pulse discharges up to 1 hour have been investigated by using ion cyclotron heating ( $\mathrm{ICH})$ with electron cyclotron heating $(\mathrm{ECH})$, or by ECH alone in the LHD.

In this paper, investigations and experimental activities on the topics of long-pulse discharges by ECH which have been performed in the LHD are introduced as follows. Section 2 describes the LHD, the ECH system and improvements in the ECH system conducted recently. Significant progress in the long-pulse discharges using EC-waves is introduced in Sec. 3. Finally, the contents of this paper are summarized in Sec. 4. 


\section{ECH system on the LHD and its upgrade}

\subsection{LHD}

The LHD is a helical device with toroidal period number $m=10$ and polarity $l=2$. The magnetic field structure with rotational transform for plasma confinement is completely generated by external superconducting magnets such as a pair of helical coils and three pairs of poloidal coils. The magnetic axis position $R_{\text {ax }}$ of LHD plasmas can be changed in the range from 3.42 to $4.1 \mathrm{~m}$. In the typical case of $R_{\mathrm{ax}}=3.6 \mathrm{~m}$, the averaged minor radius is $0.58 \mathrm{~m}$, the plasma volume is $30 \mathrm{~m}^{3}$ and the maximum magnetic field at the magnetic axis averaged in the toroidal direction, $B_{\mathrm{t}}$, is $2.85 \mathrm{~T}$.

\subsection{ECH system}

The ECH system in NIFS furnishes six working gyrotrons. The oscillation frequencies are 77 (for 3 gyrotrons), 154 (1), 84 (1) and 82.7 (1) GHz. The fundamental (2nd harmonic) resonance magnetic field of the 77 (154) $\mathrm{GHz}$ frequency is 2.75T. The 77 and $154 \mathrm{GHz}$ gyrotrons have been newly developed by collaboration with the University of Tsukuba and installed on the LHD ECH system in recent years [2, 3]. Each of the 77 and $154 \mathrm{GHz}$ gyrotrons generates more than 1.0MW port-through power. The antenna systems in two top ports $(5.5-\mathrm{U}$ for $77 \mathrm{GHz} 33$ and $9.5-\mathrm{U}$ for $77 \mathrm{GHz \# 2}$ ) and an equatorial port (2-O) are used for the 77 and $154 \mathrm{GHz}$ power injection. In the $2-\mathrm{O}$ port, two antenna systems (2-O Right for $77 \mathrm{GHz} \# 1$ and Left for $154 \mathrm{GHz})$ are installed.

\subsection{Upgrade of ECH system for long-pulse operation}

The three $77 \mathrm{GHz}$ gyrotrons were designed as over $1 \mathrm{MW}$ for pulse and $300 \mathrm{~kW}$ for continuous wave (CW) operations. In the conditioning operation using a dummy load, CW operation such as $220 \mathrm{~kW}$ for $75 \mathrm{~min}$. has been performed. However, those $77 \mathrm{GHz}$ gyrotrons have suffered gradual increase in the internal pressure of the gyrotron tubes in the case of power transmission to LHD. Stray waves inside the tube would come from reflection from the transmission line and cause local heating resulting in the pressure increase. The increase in the internal pressure prevent effective application of the $77 \mathrm{GHz}$ gyrotrons from long-pulse experiments with the pulse length of minutes-order, even with rather low LHD injection power of $\sim 100 \mathrm{~kW}$. Furthermore, the $77 \mathrm{GHz} \# 3$ gyrotron had a severe problem, which was revealed later as a distortion on its collector. The $77 \mathrm{GHz} \# 3$ gyrotron was refurbished by replacement of the collector during the maintenance period between the 16th and the 17 th experimental campaigns. Together with the collector replacement, the tube was modified, and thus furnished with a sub-window in order to remove stray waves inside the tube.

The $154 \mathrm{GHz}$ gyrotron was started to be used for the LHD experiments from the 16th experimental campaign in 2012. However, due to the lack of long-pulse conditioning operation of the tube and water-cooling channel on the power injection antenna mirrors inside the LHD vacuum vessel, it was not available for long-pulse experiments. Cooling of the antenna mirrors was improved, but insufficiently, by setting water-cooling jackets on the backsides of the antenna mirrors, and longpulse conditioning operations were performed after the 16th experimental campaign.

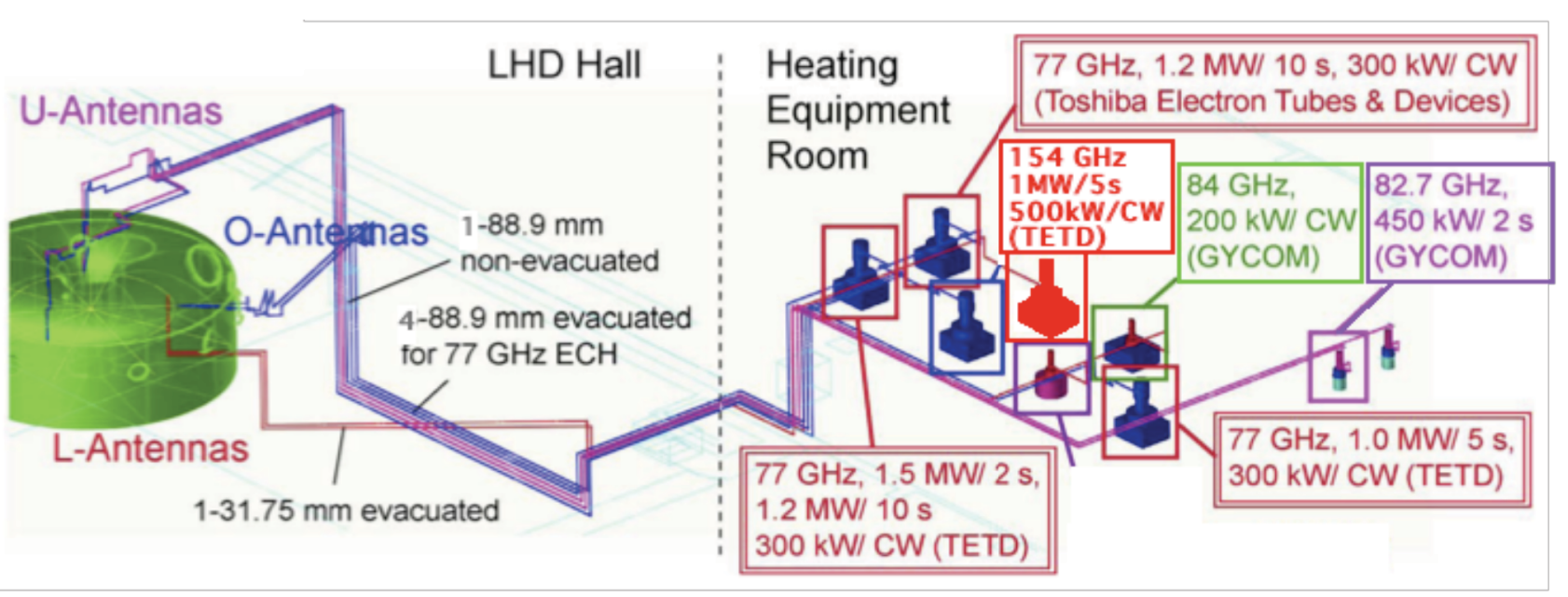

Figure 1. Schematic view of the gyrotrons in the LHD heating equipment room, transmission lines and LHD in NIFS. 


\section{Recent achievements in long-pulse discharges by EC-waves}

\subsection{Quasi-steady $30 \mathrm{~min}$. discharge}

A stable 65 min. discharge sustained with $84 \mathrm{GHz}$, $110 \mathrm{~kW}$ EC-wave was performed in 2005 [4, 5]. Due to a lack of sufficient heating power, the plasma parameter was rather low: the line average electron density $n_{\mathrm{e}_{\_} \text {ave }}$ was $\sim 0.15 \times 10^{19} \mathrm{~m}^{-3}$ and the central electron temperature $T_{\mathrm{e} 0}$ was $1.7 \mathrm{keV}$ for the $65 \mathrm{~min}$. discharge. In the LHD 16th experimental campaign in 2012, a quasi-steady 30 min. discharge by use of the upgraded ECH system: two $77 \mathrm{GHz}$ gyrotrons \#1 (110kW power injection from 2OR port), \#2 (155kW from $9.5-\mathrm{U}$ port) and an $84 \mathrm{GHz}$ gyrotron (130kW from 1.5-L port) was performed [6]. To mitigate the increase in the gyrotron internal pressure, $77 \mathrm{GHz} \# 1$ and \#2 were operated alternately with $2 \mathrm{~min}$. intervals, while the $84 \mathrm{GHz}$ gyrotron was operated continuously. The magnetic axis position $R_{\mathrm{ax}}$ and the toroidal average magnetic field on axis $B_{\mathrm{t}}$ were $3.64 \mathrm{~m}$ and $2.72 \mathrm{~T}$, respectively. The working gas for the discharge was helium.

Figure 2 shows the waveforms of $n_{\text {e_ave }}$ and the ECH injection power. Time average $n_{\mathrm{e} \_ \text {ave }}$ of $\sim 0.7 \times 10^{19} \mathrm{~m}^{-3}$, much higher than the previous $65 \mathrm{~min}$. discharge, was successfully sustained by time average ECH injection power of $260 \mathrm{~kW}$. Most of the positive spikes seen in $n_{\mathrm{e} \_ \text {ave }}$ are associated with the spikes in the signal of spectroscopic measurement of radiation from carbon (CIII). It is considered that mixings of small dusts or flakes of carbon released from the plasma facing components to the plasma would cause the temporal increases in $n_{\mathrm{e} \_a v e}[7]$.

The negative spikes in $n_{\text {e_ave }}$ are caused by $2 \mathrm{~s}$ overwraps of two power inputs from two $77 \mathrm{GHz}$ gyrotrons at every power switching timings. Temporal increases in heating power result in the temporal decreases in $n_{\mathrm{e} \_ \text {ave }}$. The central electron temperature $T_{\mathrm{e} 0}$ is $1.7 \mathrm{keV}$, and the electron temperature and density profiles of the discharge are seen in Fig. 3.

The cause of the plasma termination is not clear because of incomplete data acquisition, which sometimes occurs in long-pulse discharges. From the poloidal bolometer allay measurement, variation of peak position of the line-integrated radiation power from the plasma edge to the inner side can be seen. The source of the radiation would not be carbon. The bolometer signal increases previously to the start of increasing in the radiation of CIII at the plasma termination timing.

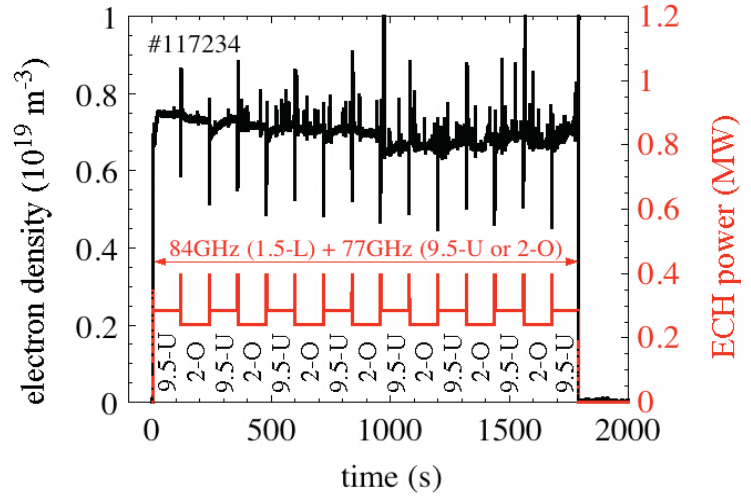

Figure 2. Waveforms of the line average electron density and the ECH injection power in the $30 \mathrm{~min}$. discharge \#117234. Two 77GHz gyrotrons were operated alternately.

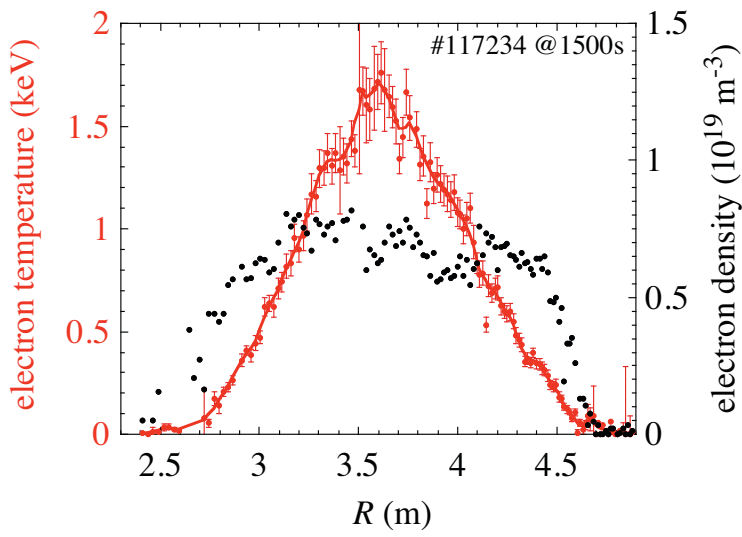

Figure 3. Electron temperature and density profiles measured at $1500 \mathrm{~s}$ with Thomson scattering in the $30 \mathrm{~min}$. discharge \#117234.

\subsection{Steady 325s discharge with ITB electron temperature profile}

After the 16th experimental campaign, conditioning operation of the $154 \mathrm{GHz}$ gyrotron for long-pulse operation was performed and completed. In the 17 th campaign in 2013 , the $154 \mathrm{GHz}$ gyrotron was applied for long-pulse experiment for the first time. However, the injection power from the $154 \mathrm{GHz}$ gyrotron should be limited to a low revel due to insufficient watercooling of the antenna mirrors for power injection.

Using the EC-waves from the $154 \mathrm{GHz}$ at $2-\mathrm{OL}$, the refurbished $77 \mathrm{GHz} \# 3$ at $5.5-\mathrm{U}$ and existing $77 \mathrm{GHz} \# 1$ at 2-OR, long-pulse plasma sustainment was attempted. The magnetic configuration was $R_{\mathrm{ax}}=3.65 \mathrm{~m}$ and $B_{\mathrm{t}}=$ 2.71T. The working gas was helium. The injection powers from the gyrotrons were $120 \mathrm{~kW}, 110 \mathrm{~kW}$, and $110 \mathrm{~kW}$, respectively, and $340 \mathrm{~kW}$ in total. Figure 4 shows the waveforms of the ECH injection power, $n_{\mathrm{e} \_a v e}$ and $T_{\mathrm{e} 0}$ of the discharge \#122257 which was sustained 
for $325 \mathrm{~s}$. The density $n_{\mathrm{e} \_ \text {ave }}$ was kept at $1.1 \times 10^{19} \mathrm{~m}^{-3}$ using a newly applied gas-feed system [8] furnished with mass-flow controllers and a new feedback control scheme for density. A temporal increase in $n_{\text {e_ave }}$ at around 250s was caused by some mishandling of the feedback control scheme.

It should be noted that the electron temperature profile with internal transport barrier (ITB) with $T_{\mathrm{e} 0}$ of $3.5 \mathrm{keV}$ was realized and sustained quite stationary during the discharge, as seen in Fig. 5, due to the increase in the injection power by applying the $154 \mathrm{GHz}$ EC-wave.

The additional sub-window on the 77GHz\#3 gyrotron worked well. The increase in the internal pressure was suppressed and almost saturated. However, by the improvement in the gyrotron itself, the next problem preventing the extension of pulse width became clear. The $325 \mathrm{~s}$ discharge was terminated due to a sudden increase in the radiation from iron. A plasma monitor using a CCD camera suggests that sparks occurring near the power injection port (5.5-U) of the $77 \mathrm{GHz} \# 3$ gyrotron would be the cause of the increase in the radiation. Responsible components on/around the power injection antenna mirrors should be identified and improved for further long-pulse discharges using $77 \mathrm{GHz} \# 3$.

\section{Conclusions}

In recent years the ECH system on the LHD has been upgraded by applying 77 and $154 \mathrm{GHz}$ gyrotrons, improving the operation scheme of the gyrotrons, and refurbishing one of the gyrotrons. The upgrade improved the plasma parameter of long-pulse discharges in LHD extensively from the former record: $n_{\mathrm{e} \_ \text {ave }}=0.15 \times 10^{19} \mathrm{~m}^{-3}$ and $T_{\mathrm{e} 0}=1.7 \mathrm{keV}$ by $110 \mathrm{~kW}$ $84 \mathrm{GHz}$ EC-wave for $65 \mathrm{~min}$. established in 2005. In the LHD 16th experimental campaign in 2012, a quasisteady $30 \mathrm{~min}$. discharge with $n_{\mathrm{e} \_ \text {ave }}=0.7 \times 10^{19} \mathrm{~m}^{-3}$ and $T_{\mathrm{e} 0}=1.7 \mathrm{keV}$ was accomplished by two alternately operated $77 \mathrm{GHz}$ gyrotrons with time average injection power of $130 \mathrm{~kW}$ and $130 \mathrm{~kW} 84 \mathrm{GHz}$ EC-wave, and thus $260 \mathrm{~kW}$ in total. In the 17 th campaign in 2013 , a high-performance plasma with internal transport barrier with $n_{\mathrm{e} \_ \text {ave }}=1.1 \times 10^{19} \mathrm{~m}^{-3}$ and $T_{\mathrm{e} 0}=3.5 \mathrm{keV}$ for $325 \mathrm{~s}$ was successfully achieved by applying the higher power of $340 \mathrm{~kW}$ : $120 \mathrm{~kW}$ from the $154 \mathrm{GHz}$ gyrotron and $110 \mathrm{~kW}$ each from two $77 \mathrm{GHz}$ gyrotrons. Suppression of the increase in the internal pressure of all the $77 \mathrm{GHz}$ gyrotrons in the case of delivering power to LHD and preventing the occurrence of the sparks inside the LHD vacuum vessel near one of the ECH power injection

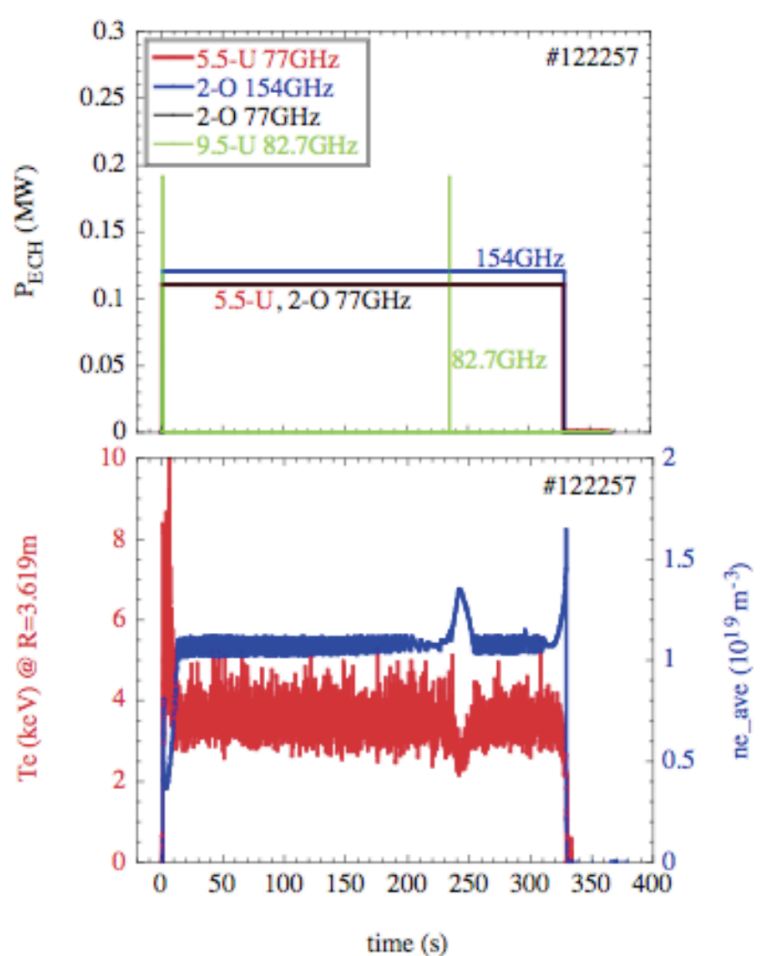

Figure 4. Waveforms of the ECH injection power, the line average electron density and the central electron temperature in the 325 s discharge \#122257.

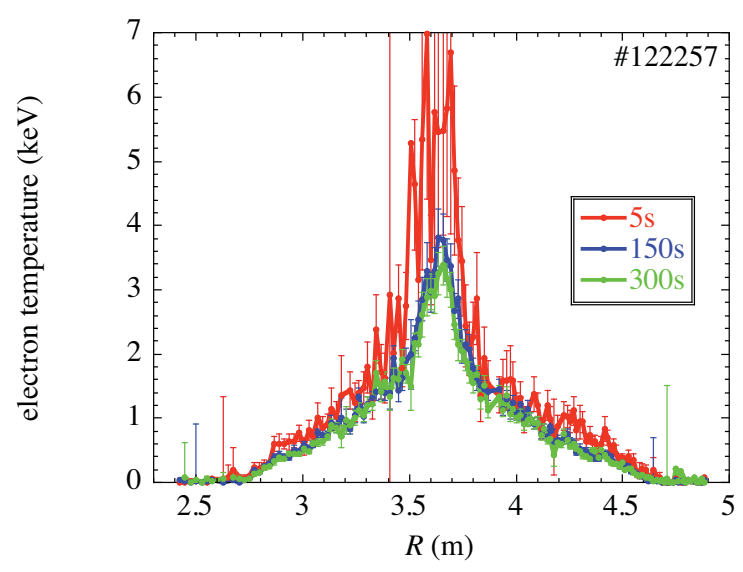

Figure 5. Electron temperature profiles measured at $5 \mathrm{~s}$, $150 \mathrm{~s}$ and $300 \mathrm{~s}$ with Thomson scattering in the $325 \mathrm{~s}$ discharge \#122257. Fine ITB profile was maintained during the discharge.

ports are the issues to be enforced for further achievements in long-pulse discharges with higher power in LHD.

\section{Acknowledgements}

The authors would like to express their thanks to NIFS staff for performing the LHD experiments. This work was supported by JSPS KAKENHI Grant Number 24561029. 


\section{References}

1. A. Komori et al., Fusion Sci. Technol. 58, 1 (2010).

2. H. Takahashi et al., Fusion Sci. Technol. 57, 19 (2010).

3. T. Shimozuma et al., Fusion Sci. Technol. 58, 530 (2010)

4. Y. Yoshimura et al., J. Phys. Conf. Ser. 25, 189 (2005)

5. Y. Yoshimura et al., Fusion Sci. Technol. 58, 551 (2010).

6. Y. Yoshimura et al., presented in Joint 19th ISHW and 16th IEA-RFP workshop, Padova, Italy (2011).

7. H. Kasahara et al., to be published in Phys. Plasmas.

8. H. Kasahara et al., to be presented in 25th IAEA Fusion Energy Conference (FEC 2014), Saint Petersburg, Russia. 